\title{
Effect of PCSK9 inhibitor on lipoprotein particles in patients with acute coronary syndromes
}

Tingting $\mathrm{Li}^{1}$, Yingyi Zhang ${ }^{2}$ and Hongliang Cong ${ }^{2^{*}}$ (])

\begin{abstract}
Background: To assess the effects of proprotein convertase subtilisin/kexin type 9 inhibitor (evolocumab) on lipoprotein particles subfractions with Nuclear Magnetic Resonance spectroscopy in patients with acute coronary syndromes.

Methods: A total of 99 consecutive patients with ACS were enrolled and assigned to either the experimental group $(n=54)$ or the control group $(n=45)$. The combination therapy of PCSK9 inhibitor (Repatha ${ }^{\circledR}, 140 \mathrm{mg}, \mathrm{q} 2 \mathrm{w}$ ) and moderate statin (Rosuvastatin, $10 \mathrm{mg}$, qn) was administered in the experimental group, with statin monotherapy (Rosuvastatin, $10 \mathrm{mg}$, qn) in the control group. The therapeutic effects on lipoprotein particle subfractions were assessed with NMR spectroscopy after 8 weeks treatment, and the achievement of LDL-C therapeutic target in both groups were analyzed.

Results: In the experimental group, after 8 weeks of evolocumab combination treatment, the concentrations of blood lipids (TC, LDL-C and its subfractions [LDL-1 to 6], VLDL-C and its subfractions [VLDL-1 to 5], IDL-C, and HDL-C), lipoprotein particles, and their subfractions [VLDL-P, IDL-P, LDL-P, and its subfractions [LDL-P1 to 6], apoB, and LP(a)] demonstrated therapeutic benefits with statistical significance $(P<0.05)$. The decrease in total LDL-P concentrations was mainly due to a decreased concentration of small-sized LDL particles (LDL-P $5+6)$, which was significantly more prominent than the decrease in medium-sized LDL-P $(L D L-P 3+4)$ and large-sized LDL-P $(L D L-P 1+2)(P<0.001)$. According to lipid control target recommended by the latest China Cholesterol Education Program Expert Consensus in 2019, after 8 weeks treatment, 96.3\% patients in the experimental group and 13.3\% in the control group had achieved the LDL-C therapeutic target $(P<0.01)$.
\end{abstract}

Conclusions: Evolocumab combination treatment for 8 weeks significantly improves the plasma lipid profiles in ACS patients, and significantly decrease the concentration of lipoprotein particles which might contribute to the pathonesis of atherosclerosis.

Keywords: PCSK9 inhibitor, Nuclear magnetic resonance spectroscopy, Lipoprotein particles, Acute coronary syndromes, Low-density lipoprotein particle

*Correspondence: hongliangcong2871@163.com; hongliangcong@126.com

${ }^{2}$ Department of Cardiology, Tianjin Chest Hospital, No. 261 Taierzhuang

South Road, Jinnan District, Tianjin, China

Full list of author information is available at the end of the article

\section{Background}

Low-density lipoprotein cholesterol (LDL-C) is the primary target of therapy in patients with acute coronary syndromes (ACS) in guidelines worldwide $[1$, 2]. Recent clinical trial evidence confirms that there is substantial incremental reduction in risk for acute 
cardiovascular events when LDL-C is reduced to very low levels $(<50 \mathrm{mg} / \mathrm{dl})$ in patients with established coronary artery disease, however, risk for events among these treated patients remained substantial [3-6]. LDL is a heterogeneous lipoprotein fraction comprising different LDL subclasses that vary in density, size and composition due to continuous remodeling of lipoproteins in the blood [7], whose chemical components and physiologic functions differ a lot from each other. LDL particles of different sizes might not play the same role in the progression of atherosclerosis [8], indicating that the subfractions of LDL particles closely correlates with their functions, which is of grander clinical significance [9-11].

Statins are the first choice for lipid-lowering agents in clinical applications, which could also effectively decrease the risk of cardiovascular diseases. Nonetheless, studies revealed that even with high-dose statins, cardiovascular events were still of elevated incidence in high-risk patients. Furthermore, some patients show poor tolerance for high-dose statin therapy. In recent years, novel lipid-lowering agents, proprotein convertase subtilisin/ kexin type 9(PCSK9) inhibitors, are receiving more attention, and impressive progresses were made in relevant studies. With the conclusion of a series of clinical trials, PCSK9 inhibitors are gradually proven to be effective in lowering blood lipid levels and preventing cardiovascular diseases [12].

The aim of this study was to evaluate the effect of statins, the traditional lipid-lowering agents, on lipoprotein particles subfractions. In addition, the effect of PCSK9 inhibitor (Repatha ${ }^{\circledR}$ ), a novel lipid-lowering agent, on lipoprotein particles subfractions will also be explored. It has been demonstrated that statins in combination with PCSK9 inhibitor can further decrease LDL-C level by $50-70 \%$ [13], bringing greater cardiovascular benefit for patients. It remains unclear whether this benefit is attributed to an overall decrease in LDL-C or the decrease in certain lipoprotein subfractions, which would be preliminary discussed in this study.

\section{Methods}

\section{Study population}

The study was approved by the Medical Ethics Committee of Tianjin Chest Hospital. Informed consents were obtained from all subjects. ACS patients who presented to the Tianjin Chest Hospital from May to December 2019 were enrolled, with the inclusion criteria as follows: (1) Informed consent obtained from the participant who voluntarily take the medications, and related documents signed; (2) Patients aged 18 years and older presenting within $72 \mathrm{~h}$ after pain onset associated with either one of the following primary diagnoses: ST-elevation myocardial infarction (STEMI), non-ST-elevation myocardial infarction (NSTEMI) or unstable angina pectoris (UAP). The diagnosis of ACS patients follows the emergency rapid diagnosis and treatment guidelines for acute coronary syndrome (2019) [14]; (3) Patients who have a clearly documented LDL-C level higher than $2.6 \mathrm{mmol} / \mathrm{l}(100 \mathrm{mg} / \mathrm{dl})$ before taking any medication or an LDL-C level higher than $1.8 \mathrm{mmol} / \mathrm{l}(70 \mathrm{mg} /$ dl) while on lipid-lowering agents. The exclusion criteria include: (1) Severe end-stage diseases, such as renal dysfunction $\left(\right.$ GFR $\left.<30 \mathrm{ml} / \mathrm{min} / 1.73 \mathrm{~m}^{2}\right)$, heart failure (left ventricular ejection fraction $<35 \%$ ), and malignant carcinoma, and liver dysfunction (liver enzymes $\geq 3$ times the upper limit of normal); (2) Systematic inflammatory disease or severe infection; (3) Thyroid disorder; (4) Pregnant or lactating women; (5) Suspected alcohol or other substance abuse, or other conditions that might impair follow-up or complicate subsequent treatment, deemed by the researcher, e.g. patients with frequent changes of workplace that might become lost on follow-up.

The 99 enrolled ACS patients were divided into two groups according to their medication: 54 in the experimental group receiving the combination therapy of PCSK9 inhibitor (Repatha ${ }^{\circledR}, 140 \mathrm{mg}, \mathrm{q} 2 \mathrm{w}$ ) and moderate statin (rosuvastatin, $10 \mathrm{mg}$, qn), and 45 in the control group receiving statin monotherapy (rosuvastatin, $10 \mathrm{mg}$, qn).

\section{Blood sample collection and lipid measurements}

At baseline and after 8 weeks treatment, the participants in the two groups were collected peripheral venous blood at the fasting and resting state in the morning for examination.

Routine blood lipid profile measurements: The participants were fasted for $8 \mathrm{~h}$. The blood was collected in a serum tube containing an inert separating gel. After the blood was fully coagulated, it was centrifuged at $3000 \mathrm{rpm}$ for $10 \mathrm{~min}$, and the supernatant was taken for testing. Lipid profile parameters (concentrations of TC, HDL-C, LDL-C, and TG) were measured using Roche c701 automated clinical chemistry analyzer. Apolipoprotein A1 (Apo-A1) and Apolipoprotein B (Apo-B) were measured by immunoturbidimetric methods. Plasma Lp(a) levels were determined by means of latex enhanced immuno-turbidimetry.

Nuclear magnetic resonance (NMR) spectroscopy measurements: $4 \mathrm{ml}$ of the participant's whole blood (fasting for $8 \mathrm{~h}$, BD blood vessels containing EDTA-K2 anticoagulant) was collected, centrifuged at $1700 \mathrm{~g}$ for $13 \mathrm{~min}$, and transferred the upper plasma into the cryopreserved tube, and then frozen and stored in aliquots at $-80{ }^{\circ} \mathrm{C}$. During the test, the samples were taken out of the refrigerator, and after thawing completely, $400 \mu \mathrm{l}$ plasma was taken and mixed with NMRS lipid buffer 
(Bruker Biospin, USA) 1:1, fully mixed, and then placed in a $5 \mathrm{~mm}$ NMR tube, and loaded into an automatic sample injector for testing [15].

Aliquots were measured blinded to patients' data by means of numbered codes and then merged with the clinical data set.

\section{Nuclear magnetic resonance (NMR) spectroscopy methods and testing program}

According to the standard operating procedure of AVANCE IVDr magnetic resonance spectrometer system (Bruker Biospin) [16, 17]. The spectra were normalized to the same quantitative scale using Bruker's QuantRef manager within TopSpin which is based on the PULCON method; hence, the spectral intensity is normalized to proton concentration in units of millimoles per liter. For data analysis, the study selected the commercial Bruker IVDr LIpoprotein Subclass Analysis (B.I.-LISA) method [18] as lipoprotein distribution prediction method, which used a PLS-2 regression model as the algorithm for spectral deconvolution [19]. This model provides information on main lipoprotein classes, including very low density lipoprotein (VLDL), intermediate density lipoprotein (IDL), LDL, and high density lipoprotein (HDL), as well as the five VLDL subfractions (VLDL-1 to VLDL-5), six LDL subfractions (LDL-1 to LDL-6), and four HDL subfractions (HDL-1 to HDL-4). Subfractions were sorted according to their increasing density and decreasing size in ascending order, respectively.

\section{Statistical analyses}

Statistical analyses were performed by SPSS 22.0. Basic parameters of descriptive statistics for the analysed continuous variables are showed as a mean and standard deviation (SD) for normal distribution or as a median of the first and third quartiles (Q1-Q3) for a distribution other than normal. Comparisons of groups regarding continuous variables are tested with the $t$ Student test (for a normal distribution of variables) or with the Mann-Whitney U test for nonnormal distributions. The categorical variables were presented as numbers (percentages), and comparisons between groups were conducted with Chi-square test. The concentrations of large-size LDL-P, medium-size LDL-P and small-size LDL-P were compared using ANOVA. Associations were examined by Pearson's correlational analyses. The interval of two-sided $P<0.05$ was considered statistically significant.

\section{Results}

\section{Baseline data}

The baseline data of the two groups was shown in Table 1 .
Demographic data, clinical characteristics, baseline blood lipid profiles, and lipoprotein particle concentrations of the 99 participants were shown in Table 1.63.0\% and $66.7 \%$ were males in the experimental group and control group, respectively; while $27.8 \%$ and $22.2 \%$ were diabetic, respectively. No significant difference was found in age, gender, statin use, comorbidities including hypertension, stroke history, dislipidemia, diabetes mellitus, MI history, prior PCI or CABG, high waist circumference and body mass index (BMI) between the two groups $(P>0.05)$. The comparisons of the prevalence of STEMI, NSTEMI, and UAP revealed no significant difference $(P>0.05)$. At baseline, the median and interquartile range of LP(a) levels in the two groups were $73.1(13.7,102.3)$ and $32.7(8.2,49.1)$, respectively, significantly higher in the experimental group with a $P$ value of 0.032 . Other baseline lipid profiles and lipoprotein particle concentrations were comparable between the two groups. The LDL-C concentrations in the experimental and control groups were $123.7 \pm 32.3 \mathrm{mg} / \mathrm{dl}$ and $103.7 \pm 28.1 \mathrm{mg} /$ $\mathrm{dl}$, respectively; and LDL-P concentrations were $1573.9 \pm 375.3 \mathrm{nmol} / \mathrm{l}$ and $1317.8 \pm 337.8 \mathrm{nmol} / \mathrm{l}$, respectively. Other baseline characteristics of blood lipid profiles and lipoprotein particles were shown in Table 1.

\section{Correlations between NMR spectroscopy and enzymatic method in the measurement of lipid parameters}

Pearson correlation analyses were performed to assess the consistency between the NMR spectroscopy and enzymatic method measurements, in terms of the six items that they had in common (triglycerides [TG], total cholesterol [TC], LDL-C, high-density lipoprotein [HDLC], Apo-A1, and Apo-B). The correlation coefficient was between 0.839 and 0.912 , indicating close correlation between the two sets of measurement (Table 2).

\section{Changes in lipid profiles after treatment in the two groups}

In the experimental group, after a combined treatment of evolocumab and moderate statins for 8 weeks, benefits in LDL-C concentrations and other blood lipids parameters were revealed with statistical significance $(P<0.05)$. TC, LDL-C and its subfractions (LDL-1 to -6), VLDL-C and its subfractions (VLDL- 1 to -5 ), and IDL-C significantly decreased compared to baseline $(P<0.001)$. The decrease in total LDL-C concentrations was mainly due to a decreased concentration of small-sized LDL particles (LDL 5+6). In contrast, a significant increase in HDL-C concentrations was observed $(P<0.05)$, which could be mainly attributed to an increase in small-sized HDL particles (HDL $3+4$ ). The concentrations of TC, LDL$\mathrm{C}$, VLDL-C and IDL-C were reduced by $48.4 \%, 65.5 \%$, $26.3 \%$, and $62.0 \%$, respectively, while the HDL-C concentrations was increased by $7.6 \%$, compared to baseline. 
Table1 Baseline clinical charateristics of the two groups and the comparisons of baseline blood lipid profiles and lipoprotein particles between them

\begin{tabular}{|c|c|c|}
\hline Variable & PCSK9i + statins $(n=54)$ & Statins $(n=45)$ \\
\hline Age (years), mean $\pm S D$ & $60.6 \pm 10.1$ & $58.6 \pm 10.6$ \\
\hline Men, n (\%) & $34(63.0 \%)$ & $30(66.7 \%)$ \\
\hline Hypertension, n (\%) & $32(59.3 \%)$ & $26(57.8 \%)$ \\
\hline Diabetes, n (\%) & $15(27.8 \%)$ & $10(22.2 \%)$ \\
\hline Stroke history, n (\%) & $6(11.1 \%)$ & $5(11.1 \%)$ \\
\hline Dislipidemia, n (\%) & $25(46.3 \%)$ & $20(44.4 \%)$ \\
\hline MI history, n (\%) & $11(20.1 \%)$ & $10(22.2 \%)$ \\
\hline Prior PCI or $\mathrm{CABG}, \mathrm{n}(\%)$ & $20(37.0 \%)$ & $18(40.0 \%)$ \\
\hline Family history of CAD, $\mathrm{n}(\%)$ & $17(31.5 \%)$ & 13(28.9\%) \\
\hline Smoking, n (\%) & $22(41.1 \%)$ & $23(51.1 \%)$ \\
\hline Drinking, n (\%) & $20(37.0 \%)$ & $16(35.6 \%)$ \\
\hline $\mathrm{BMI}\left(\mathrm{kg} / \mathrm{m}^{2}\right)$, mean $\pm \mathrm{SD}$ & $26 \pm 5.0$ & $27 \pm 5.4$ \\
\hline High waist circumference (women $>88 \mathrm{~cm}$, men $>102 \mathrm{~cm}$ ), $\mathrm{n}(\%)$ & $14(25.9 \%)$ & 13(28.9\%) \\
\hline Statin use, $n(\%)$ & $21(38.9 \%)$ & $17(37.7 \%)$ \\
\hline \multicolumn{3}{|l|}{ ACS type, $n(\%)$} \\
\hline STEMI & $10(18.5 \%)$ & $12(26.7 \%)$ \\
\hline NSTEMI & $24(44.4 \%)$ & $20(44.4 \%)$ \\
\hline UAP & $20(37.0 \%)$ & 13(28.9\%) \\
\hline Total cholesterol $(\mathrm{mg} / \mathrm{dl})$, mean \pm SD & $214.2 \pm 42.4$ & $189.7 \pm 36.5$ \\
\hline $\mathrm{LDL}-\mathrm{C}(\mathrm{mg} / \mathrm{dl})$, mean $\pm S D$ & $123.7 \pm 32.3$ & $103.7 \pm 28.1$ \\
\hline $\mathrm{IDL}-\mathrm{C}(\mathrm{mg} / \mathrm{dl})$, mean $\pm \mathrm{SD}$ & $15.5 \pm 9.1$ & $13.6 \pm 7.6$ \\
\hline $\mathrm{VLDL}-\mathrm{C}(\mathrm{mg} / \mathrm{dl})$, mean $\pm \mathrm{SD}$ & $31.9 \pm 17.9$ & $25.3 \pm 15.5$ \\
\hline $\mathrm{HDL}-\mathrm{C}(\mathrm{mg} / \mathrm{dl})$, mean $\pm \mathrm{SD}$ & $43.9 \pm 7.8$ & $44.3 \pm 9.4$ \\
\hline Lp(a) (nmol/l), median (Q1, Q3) & $73.1(13.7,102.3)$ & $32.7(8.2,49.1)$ \\
\hline ApoB $(m g / d l)$, mean $\pm S D$ & $108.7 \pm 24.2$ & $93.0 \pm 20.8$ \\
\hline LDL-P total $(\mathrm{nmol} / \mathrm{l})$, mean \pm SD & $1573.9 \pm 375.3$ & $1317.8 \pm 337.8$ \\
\hline VLDL-P $(n m o l / I)$, mean $\pm S D$ & $237.5 \pm 106.0$ & $182.8 \pm 89.2$ \\
\hline $\mathrm{IDL}-\mathrm{P}(\mathrm{nmol} / \mathrm{l})$, mean $\pm \mathrm{SD}$ & $91.0 \pm 47.7$ & $88.6 \pm 43.3$ \\
\hline
\end{tabular}

PCI percutaneous coronary intervention, CABG coronary artery bypass graft, BMI body mass index, STEMI stsegment elevation myocardial infarction, NSTEMI non-STsegment elevation myocardial infarction, UAP unstable angina pectoris, $L D L-C$ low-density lipoprotein cholesterol, IDL- $C$ intermediate-density lipoprotein, $V L D L-C$ very low-density lipoprotein cholesterol, $H D L-C$ high-density lipoprotein cholesterol, $L p(a)$ Lipoprotein a, $A p o B$ Apolipoprotein B, $L D L-P$ low-density lipoprotein particle concentration, VLDL-P very low-density lipoprotein particle concentration, IDL-P intermediate-density lipoprotein particle concentration, $Q 1, Q 3$ fifirst and third quartiles, SD standard deviation

Table 2 Pearson coefficient of correlation in lipid parameters measured by NMR spectroscopy and enzymatic method

\begin{tabular}{lll}
\hline & R values & P values \\
\hline TG & 0.849 & 0.000 \\
TC & 0.912 & 0.000 \\
HDL-C & 0.858 & 0.000 \\
LDL-C & 0.839 & 0.000 \\
Apoa1 & 0.865 & 0.000 \\
ApoB & 0.854 & 0.000 \\
\hline
\end{tabular}

After 8 weeks of single moderate statins treatment in the control group, the concentrations of TC, IDL-C, and LDL-C significantly decreased by $16.9 \%, 20.7 \%$, and $18.1 \%$, respectively $(P<0.05)$. But the concentrations of VLDL-C and HDL-C did not decrease significantly after treatment $(P>0.05)$.

After 8 weeks treatment, the absolute reduction in the concentrations of TC, LDL-C, VLDL-C, and IDL-C in the experimental and control groups were $(14.7 \pm 15.4$ vs. $5.2 \pm 12.9),(81.6 \pm 28.7$ vs. $22.9 \pm 25.1)$, $(14.7 \pm 15.4$ vs. $5.2 \pm 12.9)$, and $(10.0 \pm 7.9$ vs. $4.3 \pm 6.4)$, respectively, with significant differences between the two groups $(P<0.05)$. 


\section{Changes in lipoprotein particle concentrations after treatment in the two groups}

Changes in lipoprotein particle concentrations were presented in Table 3. After 8 weeks of combined treatment of evolocumab and moderate statins, statistically significant benefits in the concentrations of LDL-P and other lipoprotein particle concentrations were observed $(P<0.05)$. VLDL-P, IDL-P, LDL-P and its subfractions (LDL-P1 to 6), ApoB and LP(a) all decreased compared to baseline $(P<0.001)$. The concentration of total LDL-P before and after treatment was $1573.9 \pm 375.3$ and $463.6 \pm 246.7$ respectively, showing a reduction of $71.1 \%(P<0.001)$. The decrease in total LDL-P concentrations was mainly due to a decreased concentration of small-sized LDL particles (LDL 5+6). In this study, LDL-P were further classified into large (LDL-P1 +2), medium (LDL-P3+4) and small LDL-P (LDL-P5+6) by the size of the particles. The concentrations of smallsized LDL-P decreased by $76.8 \%$, with a significantly larger extent than medium-sized and large-sized LDL-P
$(P<0.001)$. The concentrations of VLDL-P, IDL-P, LP(a), and apoB decreased by $20 \%, 53.3 \%, 21 \%$, and $60.9 \%$, respectively, while the size of LDL-P in the experimental group increased by $3.6 \%(P<0.001)$, as compared to an insignificant change in the control group.

In the control group, after 8 weeks of statin monotherapy, IDL-P, LDL-P, and Apo-B concentrations significantly lowered compared to baseline $(P<0.05)$, but no significant change was found in VLDL-P concentration $(P>0.05)$. The concentrations of IDL-P, LDL-P and ApoB decreased by $24.5 \%, 21.7 \%$, and $20.8 \%$ compared to baseline. Comparison of LDL-P subfractions (small-sized, medium-sized and large-sized) revealed no significant difference $(P>0.05)$. LP(a) was seen no significant decrease, instead an increase in the control group.

After 8 weeks treatment, the absolute reductions in the concentrations of VLDL-P, IDL-P, LDL-P and ApoB were significantly different between the experimental and control group $(P<0.05)$.

Table 3 Absolute and relative reduction in lipid levels and lipoprotein particle concentration compared to baseline after 8 weeks treatment

\begin{tabular}{|c|c|c|c|c|c|c|}
\hline & Evolocumab & statins & & Statins & & \\
\hline & Week 8 & Change from baseline & $\begin{array}{l}\text { Percent change } \\
\text { from baseline (\%) }\end{array}$ & Week 8 & Change from baseline & $\begin{array}{l}\text { Percent change } \\
\text { from baseline } \\
(\%)\end{array}$ \\
\hline VLDL-C (mg/dl) Total & $17.2 \pm 7.3$ & $14.7 \pm 15.4$ & 26 & $20.0 \pm 12.1$ & $5.2 \pm 12.9$ & 7.9 \\
\hline VLDL1 & $6.7 \pm 4.6$ & $6.1 \pm 9.1$ & 26.3 & $6.5 \pm 4.5$ & $3.5 \pm 6.6$ & 21.6 \\
\hline $\operatorname{VLDL} 2+3$ & $2.6 \pm 1.5$ & $2.8 \pm 2.7$ & 20.4 & $3.6 \pm 2.5$ & $0.6 \pm 2.3$ & -20 \\
\hline $\operatorname{VLDL4}+5$ & $1.9 \pm 1.3$ & $2.2 \pm 2.5$ & 40.9 & $2.9 \pm 2.3$ & $0.6 \pm 1.7$ & 2.8 \\
\hline IDL-C (mg/dl) & $5.7 \pm 4.1$ & $10.0 \pm 7.9$ & 62.0 & $9.3 \pm 4.7$ & $4.3 \pm 6.4$ & 20.7 \\
\hline LDL-C (mg/dl) Total & $43.8 \pm 21.8$ & $81.6 \pm 28.7$ & 65.3 & $80.7 \pm 14.8$ & $22.9 \pm 25.1$ & 18.1 \\
\hline $\operatorname{LDL} 1+2$ & $10.4 \pm 4.3$ & $9.6 \pm 8.5$ & 40.4 & $11.3 \pm 4.6$ & $4.2 \pm 5.7$ & 21.2 \\
\hline $\mathrm{LDL} 3+4$ & $7.0 \pm 4.9$ & $10.2 \pm 9.4$ & 50.3 & $11.1 \pm 4.5$ & $2.4 \pm 5.9$ & -20.7 \\
\hline $\operatorname{LDL} 5+6$ & $5.0 \pm 5.6$ & $21.2 \pm 12.7$ & 79.5 & $17.6 \pm 5.9$ & $5.4 \pm 7.4$ & 6.6 \\
\hline HDL-C (mg/dl) Total & $46.9 \pm 8.3$ & $-2.9 \pm 6.5$ & -7.6 & $44.8 \pm 8.3$ & $-0.4 \pm 9.0$ & -3.4 \\
\hline $\mathrm{HDL} 1+2$ & $10.2 \pm 4.4$ & $0.7 \pm 3.6$ & -2.2 & $10.5 \pm 4.2$ & $0.1 \pm 2.6$ & -2.7 \\
\hline $\mathrm{HDL} 3+4$ & $12.6 \pm 5.4$ & $-1.9 \pm 3.2$ & -82.7 & $11.3 \pm 5.3$ & $-0.6 \pm 4.1$ & 27.2 \\
\hline $\mathrm{TC}(\mathrm{mg} / \mathrm{dl})$ & $109.9 \pm 27.9$ & $106.0 \pm 37.8$ & 48.4 & $153.7 \pm 18.4$ & $35.9 \pm 33.7$ & 16.9 \\
\hline VLDL-P (nmol/l) & $158.2 \pm 56.9$ & $80.2 \pm 84.8$ & 19.1 & $165.5 \pm 80.3$ & $17.4 \pm 79.5$ & -1.2 \\
\hline IDL-P (nmol/I) & $37.4 \pm 22.1$ & $54.0 \pm 45.2$ & 53.3 & $60.7 \pm 28.8$ & $27.8 \pm 31.9$ & 24.5 \\
\hline LDL-P (nmol/l) Total & $463.6 \pm 246.7$ & $1128.9 \pm 374.6$ & 71 & $985.2 \pm 203.3$ & $332.5 \pm 32.6$ & 21.7 \\
\hline $\operatorname{LDL} 1+2$ & $113.5 \pm 40.1$ & $91.4 \pm 81.3$ & 38.33 & $120.4 \pm 45$ & $40.5 \pm 54.1$ & 14.5 \\
\hline $\mathrm{LDL} 3+4$ & $80.1 \pm 52.7$ & $119.8 \pm 98.9$ & 57.3 & $129.5 \pm 45$ & $32.5 \pm 67.3$ & -16.7 \\
\hline LDL5 + 6 & $70.9 \pm 76.8$ & $304.8 \pm 192.1$ & 76.8 & $246.4 \pm 91.8$ & $83.0 \pm 99.4$ & 13.3 \\
\hline Lp(a) (nmol/l) & $57.5(5.2,72.8)$ & $15.6(8.5,29.5)$ & 21.1 & $42.6(8.9,62.8)$ & $-13.9(-19.2,-0.3)$ & -42.5 \\
\hline ApoB & $42.3 \pm 14.8$ & $67.5 \pm 23.6$ & 60.9 & $71.5 \pm 14.0$ & $21.5 \pm 20.2$ & 20.8 \\
\hline LDL-C:HDL-C ratio & $0.9 \pm 0.4$ & $2.0 \pm 0.8$ & 67.3 & $1.9 \pm 0.5$ & $0.5 \pm 0.7$ & 18.3 \\
\hline Apo-B:Apo-A1 ratio & $0.3 \pm 0.1$ & $0.6 \pm 0.2$ & 63.3 & $0.6 \pm 0.1$ & $0.2 \pm 0.2$ & 21.4 \\
\hline LDL-P size & $20.9 \pm 0.5$ & $-0.7 \pm 0.6$ & -3.6 & $20.2 \pm 0.2$ & $0.0 \pm 0.1$ & 0.0 \\
\hline
\end{tabular}




\section{The achievement of LDL-C therapeutic target in both groups}

After 8 weeks treatment, the mean LDL-C concentration in the experimental group decreased from 124 to $44 \mathrm{mg} /$ $\mathrm{dl}$, showing a $65.3 \%$ reduction compared to the baseline, in contrast to a $18.1 \%$ reduction from 104 to $81 \mathrm{mg} / \mathrm{dl}$ in the control group. The difference in the percentage of reduction between the two groups was $47.2 \%$, with an absolute difference of LDL-C concentration of $57 \mathrm{mg} / \mathrm{dl}$.

According to the China Cholesterol Education Program (CCEP) Expert Consensus in 2019: for patients at very high risk, the target of LDL-C concentration should be lower than $55 \mathrm{mg} / \mathrm{dl}$, or at least a $50 \%$ reduction from baseline. After 8 weeks treatment, $96.3 \%$ patients in the experimental group and $13.3 \%$ in the control group had achieved the LDL-C therapeutic target $(P<0.01)$ (Fig. 1$)$.

\section{Discussion}

PCSK9 inhibitor is a novel mechanism for reducing levels of LDL-C. Evolocumab, a fully human monoclonal antibody targeting human PCSK9, inhibits the binding of PCSK9 and LDL-R. Effective lipid modification could be achieved by PCSK 9 inhibitors, manifesting as decreased serum TC, LDL-C, and non-high-density lipoprotein cholesterol, and increased HDL-C [20]. In addition, compared to statins, PCSK9 inhibitor exhibit stronger efficacy in lowering LDL-C and TC, as well as increasing HDL-C [21]. Compared to statin alone, a combination therapy of PCSK9 inhibitor and statin demonstrates more advantages in lowering LDL-C [22]. Although the effect of evolocumab on decreasing LDL-C is well characterized, little is known about its effects on lipoprotein particles subfractions in patients with ACS in Asia.

As we all know, lipoprotein particle play an important role in atherosclerosis, including LDL-P, as well as VLDL and IDL-P. The more LDL particles could increase the risk of atherosclerosis. It has been suggested that smaller, denser LDL particles are more atherogenic than larger particles [23]. Smaller denser LDL particles may be more

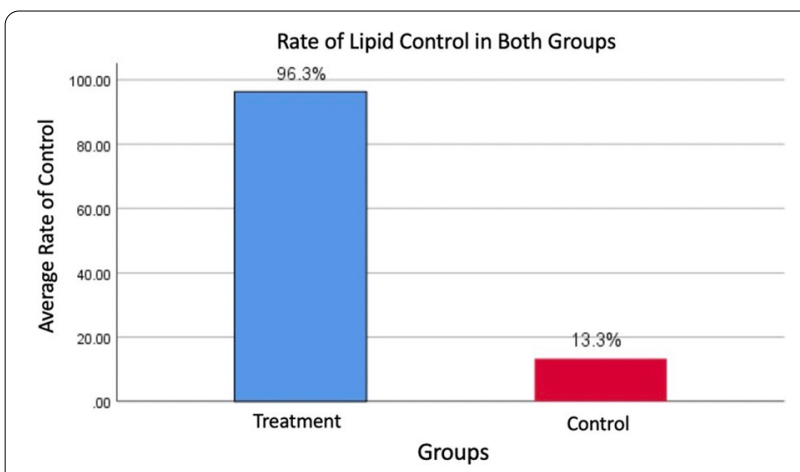

Fig. 1 The achievement of LDL-C therapeutic target in both groups atherogenic because they biophysically can more easily cross into the subendothelial space, their lipids are more prone to oxidation rendering them better substrates for macrophage scavenging, and they have lower affinity to the LDL receptor because of a change in the 3-dimensional conformation of apoprotein B [24, 25]. As remnant lipoproteins (small molecule VLDL and IDL) are also found in the atherosclerotic plaques, reduction of remnant lipoproteins is also important in addition to the reduction of LDL.

In the present study, we adopted NMR spectroscopy to assess the effects of evolocumab on blood lipid and lipoprotein particles subfractions in patients with acute coronary syndromes. Firstly, Table 1 show that baseline data were comparable between the two groups. Secondly, we analyze the correlations between NMR spectroscopy and enzymatic method in the measurement of lipid parameters. The correlation coefficient was between 0.839 and 0.912 , indicating close correlation between the two sets of measurement. Then, we compared the effects of the two treatments on blood lipid and lipoprotein particles subfractions. Both LDL-C and LDL-P were reduced significantly compared with statin monotherapy. In our study, LDL-C concentration were decreased by $65.5 \%$ in combination treatment group, which is in agreement with ODYSSEY OUTCOMES trial [26]. The observed 71\% reduction in total LDL-P concentration after 8 weeks of treatment with evolocumab combined moderate statins approximately matched the magnitude of previously reported reductions. In the Koren et al. [27] study, Alirocumab reduced mean concentrations of total LDL-P by $63.3 \%$ after 12 weeks treatment. A post hoc analysis from DESCARTES trial demonstrated that in patients receiving evolocumab, week 52 total LDL-P concentration decreased to $610 \mathrm{nmol} / \mathrm{l}$, a treatment difference of $50 \%$ versus placebo [28].

In the study, LDL-P were further classified into large (LDL-P1+2), medium (LDL-P3+4) and small LDL-P $(\mathrm{LDL}-\mathrm{P} 5+6)$ by the size of the particles. The decrease in total LDL-P concentrations was mainly due to a decreased concentration of small-sized LDL particles (LDL 5+6). The percentage reduction in small-sized LDL-P was approximately twice that of large-size LDL-P (76.8\% vs. $38.3 \%)$, which likely accounts for the increase in average LDL particle size. The results of our study was not consistent across literature [27]. The evolocumab combination treatment group substantially reduced triglyceride-enriched lipoprotein particles including VLDL$\mathrm{P}$ 、 IDL-P by $20 \%$ and $53.3 \%$, respectively. The HDL-C level was increased by $7.6 \%$. ApoB, representing the level of circulating numbers of atherogenic lipoproteins, decreased by $60.9 \%$. LP(a) decreased by $21 \%$, which is in agreement with the previous studies. 
It is well accepted that smaller size of lipoprotein particles leads to an increased risk for atherosclerosis $[29,30]$. In this study, evolocumab exhibited significant advantages in lowering the concentrations of small lipoprotein particles. We found a markedly reduction magnitude in small-size LDL (LDL5+6) and VLDL (VLDL4+5), compared to medium-size and large-size particles. Meanwhile, in evolocumab combination treatment group, the LDL-P size before and after treatment were $20.2 \pm 0.4$ and $20.9 \pm 0.5$, respectively, suggesting an increase of $3.6 \%$ in the size of lipoproteins after treatment.

2019 CCEP Expert Consensus recommended that the target of lipid control for recent ACS patients, a super high risk population of ASCVD, should achievement: an LDL-C level of less than $55 \mathrm{mg} / \mathrm{dl}$, or a reduction of LDL-C of at least $50 \%$ compared to baseline [31]. In this study, the treat-to-target rate of blood lipids with statin alone was as low as $13.3 \%$. ACS patients have a very high risk of early cardiovascular, with over $30 \%$ of cardiovascular events and mortality occurring in the first 4 days, and over 50\% occurring in the first 15 days [32]. Previous studies showed that the major cause of early ACS risks was the rupture of atherosclerotic plaques [33]. Therefore, the 2019 ESC Guidelines for the Management of Dyslipidemias recommended that: for patients who present with an ACS and whose LDL-C levels are not at goal, despite already taking a maximally tolerated statin dose and ezetimibe, the addition of a PCSK9 inhibitor early after the event (during hospitalization for the ACS event if possible) should be considered, in order to obtain earlier cardiovascular benefits [34].

\section{Study limitations}

There were some limitations in this study. First, the sample size was relatively small, and the duration was relatively short, making it difficult to evaluate the definitive clinical outcomes. Thus, future studies with larger sample size and longer follow-up period are needed to evaluate the effects of medications on clinical events and outcomes. Second, patients were not randomized. Evolocumab is still a self-funded drug in our country, the cost is more expensive. We will consider the financial situation of the patients. This could induce some bias in the selection of the sample. In conclusion, 8 weeks of PCSK9 inhibitor could significantly improve the plasma lipid profiles in ACS patients, and significantly decrease the atherogenic lipoproteins particles including LDL-P and remnant lipoproteins particles. Given the low treatto-target rate of blood lipids in ACS patients on statin monotherapy, an additional PCSK9 inhibitor is recommended to be initiated as early as possible to obtain earlier cardiovascular benefits.

\section{Conclusion}

In summary, evolocumab combination treatment for 8 weeks significantly improves the plasma lipid profiles in ACS patients, and significantly decrease the concentration of lipoprotein particles which might contribute to the pathonesis of atherosclerosis.

\begin{abstract}
Abbreviations
NMR: Nuclear magnetic resonance; ACS: Acute coronary syndromes; PCSK9: Proprotein convertase subtilisin/kexin type 9; ASCVD: Atherosclerotic cardiovascular disease; PCI: Percutaneous coronary intervention; CABG: Coronary artery bypass graft; BMI: Body mass index; STEMI: ST-segment elevation myocardial infarction; NSTEMI: Non-ST-segment elevation myocardial infarction; UAP: Unstable angina pectoris; LDL-C: Low-density lipoprotein cholesterol; IDL-C: Intermediate-density lipoprotein cholesterol; VLDL-C:Very low-density lipoprotein cholesterol; HDL-C: High-density lipoprotein cholesterol; Lp(a): Lipoprotein a; ApoB: Apolipoprotein B; LDL-P: Low-density lipoprotein particle concentration; VLDL-P:Very low-density lipoprotein particle concentration; IDL-P: Intermediate-density lipoprotein particle concentration; Q1, Q3: Fifirst and third quartiles; SD: Standard deviation.
\end{abstract}

\section{Acknowledgements}

None.

\section{Authors' contributions}

HLC made contributions to the conception and design of the study. TTL collected blood samples, analyzed the data, wrote the manuscript and drew the figures; YYZ also analyzed some data and collected the information of the subjects. All authors have read and approved the final manuscript.

\section{Funding}

None.

\section{Availability of data and materials}

The datasets used and/or analysed during the current study are available from the corresponding author on reasonable request.

\section{Ethics approval and consent to participate}

This study was approved by the Medical Ethics Committee of Tianjin Chest Hospital. Informed consents were obtained from all subjects. All patients provided written informed consent.

Consent for publication

Not applicable.

Competing interests

The authors declare that they have no competing interests.

\section{Author details}

${ }^{1}$ Tianjin Medical University, Department of Cardiology, Tianjin Chest Hospital, Tianjin, China. ${ }^{2}$ Department of Cardiology, Tianjin Chest Hospital, No. 261 Taierzhuang South Road, Jinnan District, Tianjin, China.

Received: 23 September 2020 Accepted: 15 December 2020 Published online: 07 January 2021

\section{References}

1. European Association for Cardiovascular Prevention \& Rehabilitation, Reiner Z, Catapano AL, De Backer G, Graham I, Taskinen MR, Wiklund $\mathrm{O}$, Agewall S, Alegria E, Chapman MJ, Durrington P, et al. ESC/EAS Guidelines for the management of dyslipidaemias: the Task Force for the management of dyslipidaemias of the European Society of Cardiology (ESC) and the European Atherosclerosis Society (EAS). Eur Heart J. 2011;32(14):1769-818. 
2. Stone NJ, Robinson JG, Lichtenstein AH, Bairey Merz CN, Blum CB, Eckel RH, Goldberg AC, Gordon D, Levy D, Lloyd-Jones DM, et al. 2013 ACC/ AHA guideline on the treatment of blood cholesterol to reduce atherosclerotic cardiovascular risk in adults: a report of the American College of Cardiology/American Heart Association Task Force on Practice Guidelines. Circulation. 2014;129(25 Suppl 2):S1-45.

3. Otvos JD, Mora S, Shalaurova I, Greenland P, Mackey RH, Goff DC Jr. Clinical implications of discordance between low-density lipoprotein cholesterol and particle number. J Clin Lipidol. 2011a;5(2):105-13.

4. Sabatine MS, Giugliano RP, Wiviott SD, Raal FJ, Blom DJ, Robinson J, Ballantyne CM, Somaratne R, Legg J, Wasserman SM, et al. Efficacy and safety of evolocumab in reducing lipids and cardiovascular events. N Engl J Med. 2015;372(16):1500-9.

5. Cannon CP, Blazing MA, Giugliano RP, McCagg A, White JA, Theroux P, Darius H, Lewis BS, Ophuis TO, Jukema JW, et al. Ezetimibe added to statin therapy after acute coronary syndromes. N Engl J Med. 2015;372(25):2387-97.

6. Cromwell WC, Otvos JD, Keyes MJ, Pencina MJ, Sullivan L, Vasan RS, Wilson PW, D'Agostino RB. LDL particle number and risk of future cardiovascular disease in the Framingham Offspring Study_-implications for LDL Management. J Clin Lipidol. 2007;1 (6):583-92.

7. Langlois MR, Chapman MJ, Cobbaert C, Mora S, Remaley AT, Ros E, Watts GF, Borén J, Baum H, Bruckert E, et al. European Atherosclerosis Society (EAS) and the European Federation of Clinical Chemistry and Laboratory Medicine (EFLM) Joint Consensus Initiative. Quantifying Atherogenic Lipoproteins: Current and Future Challenges in the Era of Personalized Medicine and Very Low Concentrations of LDL Cholesterol. A Consensus Statement from EAS and EFLM. Clin Chem. 2018;64(7):1006-33.

8. Goliasch G, Oravec S, Blessberger H, Dostal E, Hoke M, Wojta J, Schillinger M, Huber K, Maurer G, Wiesbauer F. Relative importance of different lipid risk factors for the development of myocardial infarction at a very young age ( $\leq 40$ years of age). Eur J Clin Invest. 2012;42(6):631-6.

9. AACC Lipoproteins and Vascular Diseases Division Working Group on Best Practices, Cole TG, Contois JH, Csako G, McConnell JP, Remaley AT, Devaraj S, Hoefner DM, Mallory T, Sethi AA, Warnick GR. Association of apolipoprotein $B$ and nuclear magnetic resonance spectroscopy-derived LDL particle number with outcomes in 25 clinical studies: assessment by the AACC Lipoprotein and Vascular Diseases Division Working Group on Best Practices. Clin Chem. 2013;59(5):752-70.

10. Mora S, Buring JE, Ridker PM. Discordance of low-density lipoprotein (LDL) cholesterol with alternative LDL-related measures and future coronary events. Circulation. 2014;129:553-61.

11. Otvos JD, Mora S, Shalaurova I, Greenland P, Mackey RH, Goff DC Jr. Clinical implications of discordance between low-density lipoprotein cholesterol and particle number. J Clin Lipidol. 201 1b;5:105-13.

12. Sabatine MS, Giugliano RP, Keech AC, Honarpour N, Wiviott SD, Murphy SA, Kuder JF, Wang H, Liu T, Wasserman SM, et al. FOURIER Steering committee and investigators: evolocumab and clinical outcomes in patients with cardiovascular disease. N Engl J Med. 2017;376(18):1713-22.

13. Nicholls SJ, Puri R, Anderson T, Ballantyne CM, Cho L, Kastelein JJ, Koenig W, Somaratne R, Kassahun H, Yang J, et al. Effect of evolocumab on progression of coronary disease in statin-treated patients: the GLAGOV randomized clinical trial. JAMA. 2016;316(22):2373-84.

14. Zhang XC, Yu XZ, Chen FY, Zhu HD. Emergency rapid diagnosis and treatment guidelines for acute coronary syndrome (2019). J Clin Emerg. 2019;20(04):253-62.

15. Jeyarajah EJ, Cromwell WC, Otvos JD. Lipoprotein particle analysis by nuclear magnetic resonance spectroscopy. Clin Lab Med. 2006;26:847-70.

16. Jiménez B, Holmes E, Heude C, Tolson RF, Harvey N, Lodge SL, Chetwynd AJ, Cannet C, Fang F, Pearce JTM, et al. Quantitative lipoprotein subclass and low molecular weight metabolite analysis in human serum and plasma by $1 \mathrm{H}$ NMR spectroscopy in a multilaboratory trial. Anal Chem. 2018;90(20):11962-71.

17. Dona AC, Jiménez B, Schäfer H, Humpfer E, Spraul M, Lewis MR, Pearce JT, Holmes E, Lindon JC, Nicholson JK. Precision high-throughput proton NMR spectroscopy of human urine, serum, and plasma for large-scale metabolic phenotyping. Anal Chem. 2014;86(19):9887-94.

18. Harbaum L, Ghataorhe P, Wharton J, Jiménez B, Howard LSG, Gibbs JSR, Nicholson JK, Rhodes CJ, Wilkins MR. Reduced plasma levels of small HDL particles transporting fibrinolytic proteins in pulmonary arterial hypertension. Thorax. 2019;74(4):380-9.
19. Okazaki M, Usui S, Ishigami M, Sakai N, Nakamura T, Matsuzawa Y, Yamashita S. Identification of unique lipoprotein subclasses for visceral obesity by component analysis of cholesterol profile in high-performance liquid chromatography. Arterioscler Thromb Vasc Biol. 2005;25(3):578-84.

20. Blom DJ, Hala T, Bolognese M, Lillestol MJ, Toth PD, Burgess L, Ceska R, Roth E, Koren MJ, Ballantyne CM, et al. DESCARTES Investigators. A 52-week placebo-controlled trial of evolocumab in hyperlipidemia. N Engl J Med. 2014;370(19):1809-19.

21. Roth EM, Taskinen MR, Ginsberg HN, Kastelein JJ, Colhoun HM, Robinson JG, Merlet L, Pordy R, Baccara-Dinet MT. Monotherapy with the PCSK9 inhibitor alirocumab versus ezetimibe in patients with hypercholesterolemia: results of a 24 week, double-blind, randomized Phase 3 trial. Int J Cardiol. 2014;176(1):55-61.

22. Koren MJ, Lundqvist P, Bolognese M, Neutel JM, Monsalvo ML, Yang J, Kim JB, Scott R, Wasserman SM, Bays H, MENDEL-2 Investigators. Anti-PCSK9 monotherapy for hypercholesterolemia: the MENDEL-2 randomized, controlled phase III clinical trial of evolocumab. J Am Coll Cardiol. 2014;63(23):2531-40.

23. Lamarche B, Tchernof A, Moorjani S, Cantin B, Dagenais GR, Lupien PJ, Després JP. Small, dense low-density lipoprotein particles as a predictor of the risk of ischemic heart disease in men. Prospective results from the Québec Cardiovascular Study. Circulation. 1997;95(1):69-75.

24. Galeano NF, Al-Haideri M, Keyserman F, Rumsey SC, Deckelbaum RJ. Small dense low density lipoprotein has increased affinity for LDL receptorindependent cell surface binding sites: a potential mechanism for increased atherogenicity. J Lipid Res. 1998;39:1263-73.

25. Toft-Petersen AP, Tilsted HH, Aarøe J, Rasmussen K, Christensen T, Griffin BA, Aardestrup IV, Andreasen A, Schmidt EB. Small dense LDL particles-a predictor of coronary artery disease evaluated by invasive and CT-based techniques: a case-control study. Lipids Health Dis. 2011;10:21.

26. Schwartz GG, Steg PG, Szarek M, Bhatt DL, Bittner VA, Diaz R, Edelberg JM, Goodman SG, Hanotin C, Harrington RA, et al. Odyssey Outcomes Committees and Investigators. Alirocumab and cardiovascular outcomes after acute coronary syndrome. N Engl J Med. 2018;379(22):2097-107.

27. Koren MJ, Kereiakes D, Pourfarzib R, Winegar D, Banerjee P, Hamon S, Hanotin C, McKenney JM. Effect of PCSK9 inhibition by Alirocumab on lipoprotein particle concentrations determined by nuclear magnetic resonance spectroscopy. J Am Heart Assoc. 2015;4(11):e002224.

28. Toth PP, Sattar N, Blom DJ, Martin SS, Jones SR, Monsalvo ML, Elliott M, Davis M, Somaratne R, Preiss D. Effect of Evolocumab on lipoprotein particles. Am J Cardiol. 2018;121(3):308-14.

29. Krauss RM. All low-density lipoprotein particles are not created equal. Arterioscler Thromb Vasc Biol. 2014;34:959-61.

30. Mora S, Caulfield MP, Wohlgemuth J, Chen Z, Superko HR, Rowland CM, Glynn RJ, Ridker PM, Krauss RM. Atherogenic lipoprotein subfractions determined by ion mobility and first cardiovascular events after random allocation to high-intensity statin or Placebo: the justification for the use of statins in prevention: an Intervention Trial Evaluating Rosuvastatin (JUPITER) Trial. Circulation. 2015;132(23):2220-9.

31. China Cholesterol Education Program Working committee. 2019 Expert recommendations of the China Cholesterol Education Program (CCEP) for lipid-lowering therapy to reduce cardiovascular events. Chin J Internal Med. 2020;1:18-22.

32. Fox KA, Anderson FA Jr, Goodman SG, Steg PG, Pieper K, Quill A, Gore JM, GRACE Investigators. Time course of events in acute coronary syndromes: implications for clinical practice from the GRACE registry. Nat Clin Pract Cardiovasc Med. 2008:5(9):580-9.

33. Gallone G, Baldetti L, Pagnesi M, Latib A, Colombo A, Libby P, Giannini F. Medical therapy for long-term prevention of atherothrombosis following an acute coronary syndrome: JACC State-of-the-Art Review. J Am Coll Cardiol. 2018;72:2886-903.

34. Authors/Task Force Members, ESC Committee for Practice Guidelines (CPG), ESC National Cardiac Societies. ESC/EAS guidelines for the management of dyslipidaemias: lipid modification to reduce cardiovascular risk. Atherosclerosis. 2019;2019(290):140-205.

\section{Publisher's Note}

Springer Nature remains neutral with regard to jurisdictional claims in published maps and institutional affiliations. 